\title{
MORE ON M. E. RUDIN'S DOWKER SPACE
}

\author{
KLAAS PIETER HART
}

ABSTRACT. It is shown that M. E. Rudin's Dowker space is finitely-fully normal and orthocompact, thus answering questions of Mansfield and Scott.

0. Introduction. In [Ma] Mansfield defined the notions of $\kappa$-full normality and finite-full normality. One of the questions he raised was, whether there exists a finitely-fully normal space which is not an $\omega_{0}$-fully normal space.

In [Sc] Scott asked whether M. E. Rudin's Dowker space [Ru] is orthocompact. We answer both questions simultaneously by showing that the above-mentioned space is both finitely-fully normal and orthocompact. Mansfield's question is hereby answered since in $\left[\mathbf{M a}\right.$ ] he showed that almost $\omega_{0}$-fully normal spaces are countably paracompact. Almost $\kappa$-full normality will not be defined here; it suffices to know that it is weaker than $\kappa$-full normality.

\section{Definitions and preliminaries.}

$1.0 \kappa$-full normality and orthocompactness. Let $Y$ be a topological space, $\mathscr{U}$ an open cover of $Y$ and $\kappa \geqslant 2$ a cardinal. An open cover $\mathcal{V}$ is said to be a $\kappa$-star (finite-star) refinement of $\mathcal{U}$ if for all $\mathcal{V}^{\prime} \subseteq \mathcal{V}$ with $\left|\mathcal{V}^{\prime}\right| \leqslant \kappa\left(\mathcal{V}^{\prime}\right.$ finite) and $\cap \mathcal{V}^{\prime} \neq \varnothing$ there is a $U \in \mathscr{Q}$ with $\cup \mathcal{V}^{\prime} \subseteq U$, and $\mathcal{V}$ is a $Q$-refinement of $\mathcal{Q}$ if $\mathcal{V}$ refines $\mathscr{U}$ and $\cap \mathcal{V}^{\prime}$ is open for all $\mathcal{V}^{\prime} \subseteq \mathcal{V}$. (Recent practice is to call $Q$-refinements interior-preserving open refinements.)

$Y$ is called $\kappa$-fully (finitely-fully) normal [Ma] if every open cover of $Y$ has a $\kappa$-star (finite-star) refinement. $Y$ is called orthocompact [Sc] if every open cover of $Y$ has a $Q$-refinement.

1.1 M. E. Rudin's Dowker space. Let $F=\prod_{n=1}^{\infty}\left(\omega_{n}+1\right)$ endowed with the box topology. Furthermore let $X^{\prime}=\left\{f \in F: \forall n \in \mathbf{N} \operatorname{cf}(f(n))>\omega_{0}\right\}$ and $X=\left\{f \in X^{\prime}\right.$ : $\left.\exists i \in \mathbf{N}: \forall n \in \mathbf{N} \operatorname{cf}(f(n))<\omega_{i}\right\}$. Then $X$ is M. E. Rudin's Dowker space [Ru].

We give an alternative description of the canonical base for $X^{\prime}$ (and $X$ ). For $f$, $g \in F$ we say

$$
\begin{aligned}
& f<g \text { if } f(n)<g(n) \text { for all } n, \\
& f \leqslant g \text { if } f(n) \leqslant g(n) \text { for all } n .
\end{aligned}
$$

For $f, g \in F$ with $f<g$ we let

$$
U_{f, g}^{\prime}=\left\{h \in X^{\prime}: f<h \leqslant g\right\}
$$

Received by the editors February 9, 1982.

1980 Mathematics Subject Classification. Primary 54D20, 54G20.

Key words and phrases. $\kappa$-fully normal, finitely-fully normal, orthocompact.

(1)1982 American Mathematical Society $0002-9939 / 82 / 0000-0212 / \$ 01.75$ 
and

$$
U_{f, g}=U_{f, g}^{\prime} \cap X
$$

Then

$$
\left\{U_{f, g}^{\left({ }^{\prime}\right)}: f, g \in F, f<g\right\}
$$

is a base for the topology of $X^{\left({ }^{\prime}\right)}$. Notice that the basic open sets are convex in the partial order $\leqslant$ on $X$, a fact we will use in the proof of Theorem 2.2.

2. The main result. In this section we prove using the results from [Ru] and [Ha] that the Dowker space $X$ is finitely-fully normal and orthocompact. First we formulate a lemma, the proof of which can be found (implicitly) in the proof in [Ru] that $X$ is collectionwise normal.

2.0 LEMMA. a. Every open cover of $X^{\prime}$ has a disjoint refinement consisting of basic open sets.

b. If $A, B \subseteq X$ are closed and disjoint then

$$
\mathrm{Cl}_{X^{\prime}} A \cap \mathrm{Cl}_{X^{\prime}} B=\varnothing .
$$

The next result is from [Ha].

2.1 Lemma. For all $n \in \mathbf{N}:\left(X^{\prime}\right)^{n}$ is homeomorphic to $X^{\prime}$, and the homeomorphism can be chosen to map $X^{n}$ onto $X$.

Now we are ready to prove the main result.

2.2 THEOREM. The space $X$ is both 2-fully normal and orthocompact.

Proof. Let $Q$ be a basic open cover of $X$. Put $U=\cup\{0 \times 0 \times 0: 0 \in \mathcal{Q}\} ; U$ is a neighborhood of $\{\langle x, x, x\rangle: x \in X\}$ in $X^{3}$. Using 2.1 and $2.0 \mathrm{~b}$ find a neighborhood $U^{\prime}$ of $\left\{\langle x, x, x\rangle: x \in X^{\prime}\right\}$ in $\left(X^{\prime}\right)^{3}$ such that $U^{\prime} \cap X^{3}=U$.

For $x \in X^{\prime} \backslash X$, choose $U_{x} \ni x$ open such that $U_{x}^{3} \subseteq U^{\prime}$.

By 2.0a let $\theta^{\prime}$ be a disjoint basic open refinement of the open cover

$$
\left\{X^{\prime} \backslash \mathrm{Cl}_{X^{\prime}}(X \backslash 0)\right\}_{0 \in \mathcal{U}} \cup\left\{U_{x}\right\}_{x \in X^{\prime} \backslash X} .
$$

Let $\theta=\left\{0^{\prime} \cap X: 0^{\prime} \in \theta^{\prime}\right\}$.

Let $0 \in \theta$ and $\{x, y, z\} \subseteq 0$.

Then $\{x, y, z\} \subseteq$ some $V \in \mathcal{Q}$ or $\{x, y, z\} \subseteq$ some $U_{p}$, but then $\langle x, y, z\rangle \in U_{p}^{3}$ $\cap X^{3} \subseteq U$, so $\langle x, y, z\rangle \in V^{3}$ for some $V \in \mathcal{Q}$ in any case. This implies that $\{x, y, z\} \subseteq V$.

For each $0 \in \theta$ define $\mho_{0}$ as follows: $0=U_{p, q}$ for some $p, q \in F$, so put $\mho_{0}=\left\{U_{p, x}: x \in 0\right\}$. Let $\mathscr{W}=\cup\left\{\mho_{0}: 0 \in \vartheta\right\}$. Then $\mathscr{W}$ is both a 2-star and a $Q$-refinement of $\mathcal{Q}$.

First, assume $U_{p, x} \cap U_{q, y} \neq \varnothing$ for some $U_{p, x}$ and $U_{q, y}$ in $थ$. Then $x$ and $y$ are elements of the same $0 \in \mathcal{O}$ and hence $p=q$. Define $p^{\prime}$ by $p^{\prime}(n)=p(n)+\omega_{1}$ $(n \in \mathbf{N})$; then $p<p^{\prime} \leqslant x, y$ and $p^{\prime} \in X$, so $p^{\prime} \in 0$.

Pick $u \in \mathcal{Q}$ such that $\left\{p^{\prime}, x, y\right\} \subseteq U$. Since $U$ is basic (and hence $\leqslant$-convex) and $U_{p, z}=\left\{t: p^{\prime} \leqslant t \leqslant z\right\}$ for $z=x, y$, it follows that $U_{p, x} \cup U_{p, y} \subseteq U$. So $\mathcal{W}$ is a 2-star

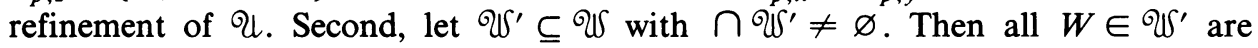


contained in the same $0 \in \mathcal{O}$, so $\mathscr{W} \mathcal{S}^{\prime}=\left\{U_{p, x}: x \in A\right\}$ for some subset $A$ of 0 , where $0=U_{p, q}$. Define $f$ by $f(n)=\min \{x(n): x \in A\}$. Then $\cap \mathcal{W}^{\prime}=U_{p, f}$ is open. So $\mathcal{W}$ is a $Q$-refinement of $Q$.

It now follows easily that $X$ is finitely-fully normal:

2.3 COROLlaRY. $X$ is finitely-fully normal.

Proof. Let $\mathcal{Q}$ be an open cover of $X$. Let $\mathcal{V}_{1}$ be a 2-star refinement of $\mathscr{Q}$, and (inductively) let $\mathcal{V}_{n+1}$ be a 2 -star refinement of $\mathcal{V}_{n}(n \in \mathbf{N})$. Since $X$ is a $P$-space ( $G_{\delta}$ 's are open) we can take the common refinement of all $\mathcal{V}_{n}$; call it $\mathfrak{V}$. Let $\mathcal{V}^{\prime} \subseteq \mathcal{V}$ be finite with $\cap \mathcal{V}^{\prime} \neq \varnothing$. Pick $n \in \mathbf{N}$ such that $2^{n} \geqslant\left|\mathcal{V}^{\prime}\right|$. Since $\mathcal{V}$ refines $\mathcal{V}_{n}$ and since $\mathcal{V}_{n}$ is a $2^{n}$-star refinement of $\mathscr{U}$, it follows that $\cup \mathcal{V}^{\prime}$ is contained in some $U \in \mathcal{Q}$.

\section{REFERENCES}

[Ha] K. P. Hart, Strong collectionwise normality and M. E. Rudin's Dowker space, Proc. Amer. Math. Soc. 83 (1981), 802-806.

[Ma] M. J. Mansfield, Some generalizations of full normality, Trans. Amer. Math. Soc. 86 (1957), 489-505.

[Ru] M. E. Rudin, A normal space $X$ for which $X \times I$ is not normal, Fund. Math. 73 (1971), 179-186.

[Sc] B. M. Scott, Toward a product theory for orthocompactness, Studies in Topology, Academic Press, New York, 1975, pp. 517-537.

Subfaculteit Wiskunde, Vrije Universiteit, De BoelelaAn 1081, 1081 HV Amsterdam, The NETHERLANDS 